\title{
Evaluating the association of social needs assessment data with cardiometabolic health status in a federally qualified community health center patient population
}

Connor Drake ${ }^{1,2^{*}}$, Tyler Lian ${ }^{1}$, Justin G. Trogdon², David Edelman³ ${ }^{3,4}$, Howard Eisenson $^{5,6}$, Morris Weinberger ${ }^{2}$, Kristin Reiter ${ }^{2}$ and Christopher M. Shea ${ }^{2}$

\begin{abstract}
Background: Health systems are increasingly using standardized social needs screening and response protocols including the Protocol for Responding to and Assessing Patients' Risks, Assets, and Experiences (PRAPARE) to improve population health and equity; despite established relationships between the social determinants of health and health outcomes, little is known about the associations between standardized social needs assessment information and patients' clinical condition.

Methods: In this cross-sectional study, we examined the relationship between social needs screening assessment data and measures of cardiometabolic clinical health from electronic health records data using two modelling approaches: a backward stepwise logistic regression and a least absolute selection and shrinkage operation (LASSO) logistic regression. Primary outcomes were dichotomized cardiometabolic measures related to obesity, hypertension, and atherosclerotic cardiovascular disease (ASCVD) 10-year risk. Nested models were built to evaluate the utility of social needs assessment data from PRAPARE for risk prediction, stratification, and population health management.
\end{abstract}

Results: Social needs related to lack of housing, unemployment, stress, access to medicine or health care, and inability to afford phone service were consistently associated with cardiometabolic risk across models. Model fit, as measured by the $c$-statistic, was poor for predicting obesity (logistic $=0.586 ;$ LASSO $=0.587$ ), moderate for stage 1 hypertension (logistic $=0.703 ; \mathrm{LASSO}=0.688$ ), and high for borderline ASCVD risk (logistic $=0.954 ; \mathrm{LASSO}=0.950$ ).

Conclusions: Associations between social needs assessment data and clinical outcomes vary by cardiometabolic condition. Social needs assessment data may be useful for prospectively identifying patients at heightened cardiometabolic risk; however, there are limits to the utility of social needs data for improving predictive performance.

Keywords: Social determinants of health, Social needs, Primary care, Predictive analytics, Electronic health record

\footnotetext{
*Correspondence: connor.drake@duke.edu

1 Department of Population Health Sciences, Duke University School

of Medicine, 215 Morris Street, Durham, NC 27701, USA

Full list of author information is available at the end of the article
}

\section{Background}

Social determinants of health (SDOH), such as access to nutritious food, transportation, employment, and stable housing, are significant drivers of health outcomes [1, 2]. For health care systems to successfully reform delivery towards value, prevention, and effective population health management, they need to assess and respond to 
social needs associated with downstream consequences of the $\mathrm{SDOH}[3,4]$. To this end, health care systems and payers are increasingly collecting population- and individual-level data on social needs, including food insecurity, unemployment, housing instability, and transportation barriers [5, 6].

In 2016, the Protocol for Responding to and Assessing Patients' Assets, Risks, and Experiences (PRAPARE) was developed by the National Association of Community Health Centers and partnering organizations as a screening tool and corresponding clinical workflow to assess and respond to patients' social needs. PRAPARE has been the most prevalent social needs assessment in the United States, increasingly used by hospitals, health systems, and health plans $[7,8]$. The PRAPARE screening assessment bridges social risk and clinical risk indicators by being embedded into electronic health record (EHR) systems and has facilitated national standards surrounding social risk data capture, reporting, and population health and care management activities [9].

Despite these features and the prevalence of PRAPARE, there is little evidence on the relationship between data from PRAPARE and clinical outcomes of interest [10]. A recent systematic review of PRAPARE and similar social needs screening assessments found little evidence to evaluate predictive validity [11]. Addressing this gap is critical as health care systems consider delivery reforms that embrace population health management and care coordination across the health and social care continuum $[4,12]$. With a better understanding of how social needs screening assessment data predicts clinical risk, health systems and payers can identify patients with health related social needs that are most predictive of poor health, provide care management to promote linkages to appropriate wraparound services and community resources to patients most likely to realize health benefits, measure the impact of interventions, manage patient panels, and inform care team composition [13]. As health systems and payers increasingly invest in collecting this information $[4,14,15]$, there is a need to evaluate the relationship between patients' social needs and their clinical risk to design tailored interventions.

This cross-sectional study examined the relationship between responses to PRAPARE and cardiometabolic clinical outcomes among patients in a federally-qualified community health center (FQHC). We utilized two approaches to examine the association between social needs assessment data and the likelihood of the following clinical indicators of cardiometabolic health status: obesity, hypertension, and atherosclerotic cardiovascular disease (ASCVD) 10-year risk. These outcomes are important because cardiometabolic disease is the leading cause of death of people in the United States, and obesity and hypertension are both modifiable risk factors [16]. Our goals were to (1) better understand the social needs and health status of a defined population and (2) evaluate PRAPARE social needs assessment data's association with cardiometabolic health status to inform risk prediction, stratification, and population health management. We hypothesized that models using social needs data from PRAPARE would have moderate performance for three cardiometabolic health outcomes.

\section{Methods \\ Study setting and data collection}

The study was conducted at a FQHC in a medium-sized city in the southeastern United States. In 2019, the FQHC saw 36,361 unique patients, $97 \%$ of whom had incomes $\leq 200 \%$ of the federal poverty level; $57 \%$ were uninsured and $93 \%$ were members of racial and/or ethnic minorities.

The FQHC began implementing PRAPARE in mid2017 in its Pediatric, Adult Medicine, and Family Medicine clinics. PRAPARE tool was fully integrated within the FQHC's EHR. The social needs assessment is administered via patient interview, and referrals to community resources or social services are made based on identified needs. Additional detail on the FQHC's clinical workflow, patient population, EHR integration, and implementation logistics are published elsewhere [17].

\section{Measures}

We obtained the data used in this analysis through a retrospective query to abstract charts of patients who had received PRAPARE as part of their primary care clinical encounter. PRAPARE includes a set of national, well-validated core measures and additional optional measures to match community priorities [18]. The core measures evaluate the following: race, ethnicity, education, employment, migrant/seasonal farm work, insurance, veteran status, income, language, material security (food, clothing, childcare, utilities, medicine/health care, phone, and other), transportation, housing status, housing stability, social integration and support, address/neighborhood, and stress. The optional measures include incarceration history, safety, refugee status, and domestic or interpersonal violence. PRAPARE aligns with existing national initiatives [19], ICD-10 clinical coding, and the Uniform Data System used by the Health Resources and Services Administration. All core and optional measures were included as independent variables except for neighborhood, incarceration history, refugee status (not consistently collected during the chart abstraction period), language (data missingness and correlation with ethnicity), and income (data quality/missingness). Sex and age were included as covariates in the analysis. 
We evaluated three cardiometabolic clinical outcomes: body mass index (BMI), systolic and diastolic blood pressure, and ASCVD 10-year risk. These outcomes were selected because of their relevance in primary care [20], data availability and integrity, and causal links to how social needs can affect clinical risk [21]. All three clinical outcomes were dichotomized: obesity was defined as $\mathrm{BMI} \geq 30$ for all patients with recorded height and weight $(\mathrm{n}=2153)$. Hypertension was defined by a stage-1 threshold using patients' systolic $(>130 \mathrm{mmHg}$ ) or diastolic (>80 $\mathrm{mmHg}$ ) blood pressure $(\mathrm{n}=2174)$ [22]. Finally, the ASCVD risk score is an estimate of the likelihood of an ASCVD event over the following 10 years and was developed to identify patients that might benefit from primary prevention [23]. Patients with a risk score $>7.5 \%$ were classified as being at risk requiring clinical intervention using statin therapy based on existing guidelines [24] Because ASCVD is not a valid cardiovascular estimate for patients younger than 40 years, they were excluded, leading to an analytic sample of 1468 patients after imputing missing cholesterol readings with a healthy value as is consistent with best practices for managing missing EHR data [25].

\section{Statistical analysis}

\section{Regression methods}

We employed two standard model selection approaches to assess the associations between PRAPARE responses and the three clinical outcomes: (1) backwards stepwise logistic regression, a parametric approach in which predictor variables are included in the model and removed individually if they were not statistically significant at a 0.157 level, a value recognized to optimize the Akaike information criteria (AIC) [26, 27]; and (2) logistic least absolute selection and shrinkage operation (LASSO) regression, a type of supervised machine learning algorithm that performs model selection by "shrinking" or penalizing variables, (i.e., setting certain coefficients to zero if they are not contributing explanatory power to the model) [28]. As a result, LASSO is designed to avoid overfitting better than regression models without a penalization function and uses a data-driven approach to select variables, minimize prediction error, maximize out-of-sample performance, and address issues with multicollinearity. We used three different LASSO models with different model selection criteria. One was based on an adaptive LASSO, one minimized the Bayesian information criteria (BIC), and one minimized AIC, which estimates the amount of information lost by using the model [29]. Model fit was similar across all three LASSO approaches, but the AIC approach consistently performed well and was selected based on performance and theory. Additional results comparing the different
LASSO approaches for all variables of interest can be found in Additional file 1.

For each outcome and regression approach, nested sequential models were built by adding social need and demographic covariates in groupings. This sequential approach highlighted changes in model performance and goodness-of-fit as PRAPARE covariates are added to the limited set of demographic characteristics usually operationalized in typical EHR. Model 1 used only age and sex; Model 2 added race and ethnicity; and Model 3 added expanded demographic covariates from the PRAPARE that can often be found in existing EHR data (e.g., household size, education, employment, insurance status). The full model, Model 4, added the remaining social needs covariates identified during screening. For each model, the backwards stepwise or LASSO approach selected variables to include. Excluded variables were also excluded in all subsequent models to enable direct comparison of the nested models.

\section{Model evaluation}

To evaluate model goodness-of-fit, a likelihood-ratio test was conducted for each successive pair of nested models. To evaluate model performance, we computed the concordance statistic (c) and assessed the statistical significance of c-statistic improvement between sequential models using a equality-of-areas test [30]. The c-statistic represents the area under the receiver operating characteristic curve, which can range from 0 to 1 . A c-statistic of 0.5 indicates that the model performs as well as random chance at classifying outcomes and 1 indicates perfect accuracy. For the stepwise logistic regression models, c-statistics were computed over the full dataset; for the LASSO regression models, c-statistics were computed over a validation dataset that contained $20 \%$ of the observations, which were excluded from model training. We hypothesized that model performance for the full PRAPARE model (Model 4) would be satisfactory across the three clinical outcomes. We defined satisfactory performance as having a c-statistic $>0.65$, the lower bound for moderate discrimination [31]. All statistical analysis was conducted in Stata version 16 [32].

\section{Results \\ Study sample}

Between May 2017 and February 2019, PRAPARE was delivered to 2192 patients, primarily those who were referred to behavioral health either as part of a primary care or stand-alone appointment (Table 1 ). The average patient age was 50 and $\sim 60 \%$ of patients were female. Almost half of patients were African American and about a third were Hispanic. Approximately one third of patients lacked a high school education 
Table 1 Description of differences between patients across clinical risk indicators

\begin{tabular}{|c|c|c|c|c|c|c|c|c|c|c|}
\hline & \multirow[t]{2}{*}{ Overall } & \multicolumn{3}{|c|}{ Obese } & \multicolumn{3}{|c|}{ High BP, Stage 1} & \multicolumn{3}{|c|}{ ASCVD Risk } \\
\hline & & - & + & $P$ value & - & + & $P$ value & - & + & $P$ value \\
\hline N & 2192 & 980 & 1173 & - & 1027 & 1147 & - & 387 & 1081 & - \\
\hline \multicolumn{11}{|l|}{ Demographics $^{\mathrm{a}}$} \\
\hline Age, year, mean & 49.6 & 49.2 & 50.2 & 0.102 & 47.9 & 54.8 & $<0.001^{*}$ & 47.4 & 58.7 & $<0.001^{*}$ \\
\hline Female & 61.8 & 53.3 & 68.8 & $<0.001^{*}$ & 63.9 & 55.6 & $<0.001^{*}$ & 79.1 & 52.5 & $<0.001^{*}$ \\
\hline \multicolumn{11}{|l|}{ Race } \\
\hline Black/African American & 49.5 & 45.5 & 52.6 & $0.001^{*}$ & 43.1 & 66.5 & $<0.001^{*}$ & 26.1 & 71.3 & $<0.001^{*}$ \\
\hline White/Caucasian & 14.0 & 15.8 & 12.4 & $0.021^{*}$ & 14.9 & 11.6 & $0.050^{*}$ & 18.1 & 14.1 & 0.058 \\
\hline Other & 26.7 & 28.5 & 25.5 & 0.120 & 30.5 & 16.5 & $<0.001^{*}$ & 55.8 & 14.6 & $<0.001^{*}$ \\
\hline Not reported/declined & 9.9 & 10.2 & 9.6 & 0.611 & 11.5 & 5.4 & $<0.001^{*}$ & - & - & - \\
\hline Hispanic/Latino & 35.1 & 36.4 & 34.4 & 0.330 & 40.7 & 19.9 & $<0.001^{*}$ & 56.8 & 12.8 & $<0.001^{*}$ \\
\hline \multicolumn{11}{|l|}{ Members per household } \\
\hline Lives alone & 27.9 & 30.1 & 26.2 & 0.059 & 25.8 & 33.7 & $0.001^{*}$ & 16.3 & 40.5 & $<0.001^{*}$ \\
\hline Two & 22.8 & 22.0 & 23.5 & 0.450 & 21.3 & 27.0 & $0.008^{*}$ & 18.3 & 26.5 & $0.002^{*}$ \\
\hline Three to four & 30.9 & 30.8 & 31.0 & 0.914 & 32.6 & 26.0 & $0.005^{*}$ & 40.3 & 22.9 & $<0.001^{*}$ \\
\hline More than five & 18.4 & 17.1 & 19.3 & 0.215 & 20.3 & 13.3 & $<0.001^{*}$ & 25.1 & 10.1 & $<0.001^{*}$ \\
\hline Migrant or seasonal work & 0.8 & 0.9 & 0.7 & 0.753 & 0.7 & 0.9 & 0.647 & 0.8 & 0.5 & 0.479 \\
\hline Military discharge & 1.6 & 1.8 & 1.4 & 0.405 & 1.7 & 1.3 & 0.576 & 0.3 & 2.9 & $0.004^{*}$ \\
\hline Uninsured & 58.4 & 60.5 & 56.7 & 0.081 & 59.7 & 55.4 & 0.081 & 68.3 & 43.6 & $<0.001^{*}$ \\
\hline Lacks high school education & 34.6 & 35.3 & 33.8 & 0.502 & 35.8 & 31.8 & 0.099 & 41.8 & 29.5 & $<0.001^{*}$ \\
\hline \multicolumn{11}{|l|}{ Work situation } \\
\hline Full-time & 24.8 & 25.0 & 25.1 & 0.959 & 27.4 & 18.0 & $<0.001^{*}$ & 34.3 & 15.5 & $<0.001^{*}$ \\
\hline Part-time & 19.6 & 20.9 & 18.5 & 0.168 & 21.3 & 14.6 & $0.001^{*}$ & 26.8 & 13.5 & $<0.001^{*}$ \\
\hline Unemployed, seeking work & 26.6 & 25.8 & 27.5 & 0.409 & 24.3 & 33.5 & $<0.001^{*}$ & 23.2 & 31.7 & $0.002^{*}$ \\
\hline Unemployed, not seeking work & 29.0 & 28.3 & 29.0 & 0.726 & 27.1 & 33.9 & $0.003^{*}$ & 15.8 & 39.3 & $<0.001^{*}$ \\
\hline \multicolumn{11}{|l|}{ Social needs ${ }^{\mathrm{a}}$} \\
\hline No housing & 19.4 & 22.3 & 16.6 & $0.001^{*}$ & 18.7 & 21.4 & 0.167 & 15.3 & 21.6 & $0.009^{*}$ \\
\hline Worried about losing housing & 14.3 & 14.7 & 14.1 & 0.721 & 14.4 & 14.1 & 0.859 & 20.2 & 12.1 & $<0.001^{*}$ \\
\hline Lacks transportation & 17.6 & 19.7 & 15.7 & $0.022^{*}$ & 17.3 & 18.2 & 0.656 & 17.7 & 17.4 & 0.890 \\
\hline Low social interaction & 37.0 & 37.3 & 36.4 & 0.658 & 36.3 & 38.6 & 0.339 & 34.4 & 37.9 & 0.237 \\
\hline High stress & 14.3 & 16.3 & 12.2 & $0.009^{*}$ & 13.8 & 15.4 & 0.354 & 17.8 & 13.0 & $0.024^{*}$ \\
\hline Feels unsafe at residence & 7.7 & 8.1 & 7.3 & 0.548 & 7.8 & 7.6 & 0.862 & 10.1 & 8.0 & 0.210 \\
\hline Afraid of partner & 3.7 & 3.4 & 3.7 & 0.675 & 4.1 & 2.6 & 0.138 & 3.6 & 2.2 & 0.149 \\
\hline \multicolumn{11}{|l|}{ Other material need } \\
\hline Food & 16.2 & 15.9 & 16.4 & 0.738 & 14.9 & 20.0 & $0.006^{*}$ & 10.6 & 20.4 & $<0.001^{*}$ \\
\hline Access to health care & 19.1 & 20.2 & 18.0 & 0.207 & 17.1 & 25.4 & $<0.001^{*}$ & 16.3 & 21.6 & $0.027^{*}$ \\
\hline Utilities & 7.3 & 7.4 & 0.7 .2 & 0.840 & 6.7 & 8.9 & 0.097 & 6.5 & 8.0 & 0.355 \\
\hline Clothing & 4.4 & 5.3 & 3.6 & 0.074 & 4.0 & 5.6 & 0.136 & 3.0 & 5.1 & 0.098 \\
\hline Child care & 1.5 & 1.4 & 1.5 & 0.783 & 1.5 & 1.3 & 0.714 & 0.3 & 0.7 & 0.370 \\
\hline Phone & 2.6 & 2.7 & 2.5 & 0.842 & 1.8 & 4.8 & $<0.001^{*}$ & 1.1 & 3.4 & $0.020^{*}$ \\
\hline Other & 7.0 & 7.5 & 6.4 & 0.345 & 7.3 & 5.9 & 0.275 & 6.8 & 6.9 & 0.925 \\
\hline
\end{tabular}

${ }^{a}$ Reported as \%, unless otherwise specified

${ }^{*} P<0.05$

and the majority were uninsured or unemployed. The most commonly-reported social needs were social isolation, barriers to health care and medicine access, lack of housing, transportation barriers, food insecurity, and high stress. Over half of patients (54.5\%) were obese, $52.8 \%$ had stage- 1 hypertension, and $73.6 \%$ had borderline 10-year ASCVD risk or higher. Since the calculation of ASCVD risk is only valid for patients age 40-79, 
the analytic subsample for the ASCVD model is smaller $(\mathrm{n}=1468)$; including a smaller proportion of Hispanic/ Latino patients ( $24.4 \%$ vs. $35.1 \%$ overall).

The presence of social needs was generally greater among patients with adverse health outcomes (Table 1). In bivariate analyses, food insecurity, lack of access to care and medicine, and inability to afford a phone plan were significantly more prevalent in patients with high blood pressure and borderline-or-higher ASCVD risk than in patients without. However, this trend was not the case for obese patients, who were more likely to have housing, had fewer transportation barriers, and lower stress.

\section{Nested models}

For both the stepwise logistic and LASSO approaches, the full models (Model 4) for all three clinical outcomes included both demographic and social need variables (Additional file 2). The number of variables retained in each model ranged between 7 and 13 for the stepwise logistic regression models and between 5 and 13 for the LASSO logistic regression models. The variables that were included in at least three models were: age, sex, race, lack of housing, unemployment, high stress, and access to health care or medicine.

In multivariable analyses, the magnitude and direction of the odds ratios were similar between the stepwise and LASSO approaches (Table 2). High stress and lack of housing were associated with decreased odds of obesity

Table 2 Estimated coefficients for final models (Model 4s)

\begin{tabular}{|c|c|c|c|c|c|c|}
\hline & \multicolumn{2}{|l|}{ Obesity } & \multicolumn{2}{|c|}{ Hypertension, stage-1 } & \multicolumn{2}{|c|}{ ASCVD, borderline } \\
\hline & Stepwise $^{a}$ & LASSO $^{b}$ & Stepwise $^{a}$ & LASSO $^{b}$ & Stepwise $^{a}$ & LASSO $^{b}$ \\
\hline Age & $1.01(1.00,1.01)$ & 1.01 & $1.04(1.03,1.05)$ & 1.04 & $1.27(1.22,1.31)$ & 1.26 \\
\hline Female & $2.08(1.71,2.54)$ & 2.13 & $0.76(0.62,0.93)$ & 0.77 & $0.10(0.06,0.16)$ & 0.10 \\
\hline \multicolumn{7}{|l|}{ Race } \\
\hline Ref: Black/African Am & & & Ref & Ref & Ref & Ref \\
\hline White/Caucasian & & & $0.50(0.36,0.68)$ & 0.44 & $0.14(0.08,0.25)$ & 0.18 \\
\hline Other & & & $0.41(0.32,0.52)$ & 0.42 & $0.13(0.06,0.31)$ & 0.16 \\
\hline Not reported/declined & & & $0.42(0.30,0.59)$ & 0.46 & & \\
\hline Hispanic/Latino & & & & 0.88 & $0.57(0.25,1.28)$ & 0.60 \\
\hline \multicolumn{7}{|l|}{ Members per household } \\
\hline Ref: One & & & Ref & Ref & & \\
\hline Two & & & $1.25(0.98,1.60)$ & 1.19 & & \\
\hline Migrant or seasonal work & & & $3.45(1.01,11.8)$ & 3.99 & & \\
\hline Military discharge & & & & 1.98 & & \\
\hline Uninsured & & & & 1.23 & $1.18(0.75,1.86)$ & \\
\hline \multicolumn{7}{|l|}{ Work situation } \\
\hline Ref: Full-time & Ref & & Ref & Ref & Ref & Ref \\
\hline Part-time & $0.79(0.62,1.02)$ & & & & & \\
\hline Unemp., seeking work & & & & & $1.77(1.10,2.85)$ & \\
\hline Unemp., not seeking work & $0.85(0.67,1.08)$ & & $0.79(0.62,1.00)$ & 0.79 & $1.63(0.93,2.85)$ & 1.73 \\
\hline No housing & $0.75(0.59,0.95)$ & 0.86 & & & & 1.09 \\
\hline Lacks transportation & $0.80(0.62,1.03)$ & 0.83 & & & & \\
\hline Low social interaction & & & & & & 1.08 \\
\hline High stress & $0.66(0.50,0.88)$ & 0.74 & & & $0.63(0.26,1.11)$ & 0.76 \\
\hline Feels unsafe at residence & & & & & $0.56(0.28,1.13)$ & \\
\hline \multicolumn{7}{|l|}{ Other material need } \\
\hline Food & & & & & $1.80(0.96,3.36)$ & 1.32 \\
\hline Access to health care & & & $1.53(1.19,1.97)$ & 1.16 & $1.67(0.97,2.87)$ & 1.17 \\
\hline Child care & & & & & & 2.71 \\
\hline Phone & & & & & $3.65(0.78,17.0)$ & 2.27 \\
\hline
\end{tabular}

a Odds ratios and 95\% confidence intervals (lower, upper) reported

${ }^{b}$ Odds ratios reported; the LASSO algorithm does not compute $P$ values 
in full models. Identified needs related to health care and medicine access were associated with increased odds of both hypertension and borderline-or-higher ASCVD risk. Lack of housing was associated with lower odds of obesity, but higher odds of borderline-or-higher ASCVD risk.

For all three clinical outcomes, the final stepwise and LASSO models performed similarly, as measured by c-statistic (Table 3 ). Model performance was poor for predicting obesity (stepwise, $\mathrm{c}=0.617$; LASSO, $\mathrm{c}=0.590$ ), moderate for hypertension (stepwise, $\mathrm{c}=0.711$; LASSO, $\mathrm{c}=0.681$ ), and high for ASCVD risk (stepwise, $\mathrm{c}=0.944$; LASSO, $\mathrm{c}=0.949$ ). The high prediction performance for ASCVD risk was expected as age, race, and sex are used to calculate the score and were included as covariates alongside the PRAPARE variables.

With each sequential model, improvements in performance were observed as covariates were added; however, improvements were not always statistically significant (Table 3). For obesity, the addition of the social needs covariates (Model 4) resulted in a statistically significant increase in the c-statistic, but only in the stepwise approach. For hypertension, only the inclusion of race and ethnicity covariates (for Model 2) was associated with a significant increase in the c-statistic for both approaches. For ASCVD risk, the addition of the social needs covariates (for Model 4) significantly increased the c-statistic for the stepwise approach; however, for the LASSO approach, the c-statistic plateaued in Models 2, 3 and 4, with a slight decrease in performance in Models 3 and 4.

Sequential models often demonstrated statistically significant improvements in goodness-of-fit (Table 3). In particular, for both regression approaches, the addition of the social needs covariates from PRAPARE in all three clinical outcomes significantly improved goodness-of-fit compared to the model that included extended demographics only.

Table 3 Performance and goodness-of-fit of nested models

\begin{tabular}{|c|c|c|c|c|c|c|}
\hline Outcome & Model & C-statistic & $95 \% \mathrm{Cl}, \mathrm{LL}$ & $95 \% \mathrm{Cl}, \mathrm{UL}$ & 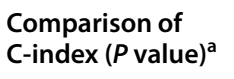 & $\begin{array}{l}\text { Likelihood-ratio } \\
\text { test }(P \text { value })^{\mathrm{a}}\end{array}$ \\
\hline \multicolumn{7}{|c|}{ Backwards stepwise logistic } \\
\hline \multirow[t]{4}{*}{ Obesity } & M1 & 0.5941 & 0.5675 & 0.6208 & - & - \\
\hline & M2 & 0.5941 & 0.5675 & 0.6208 & 1.0000 & 1.0000 \\
\hline & M3 & 0.5992 & 0.5728 & 0.6257 & 0.4163 & 0.0836 \\
\hline & M4 & 0.6173 & 0.5912 & 0.6433 & $0.0133^{*}$ & $0.0002^{*}$ \\
\hline \multirow[t]{4}{*}{ Hypertension, stage-1 } & M1 & 0.6670 & 0.6421 & 0.6920 & - & - \\
\hline & M2 & 0.7013 & 0.6771 & 0.7256 & $<0.0001^{*}$ & $<0.0001^{*}$ \\
\hline & M3 & 0.7059 & 0.6818 & 0.7299 & 0.0991 & $0.0106^{*}$ \\
\hline & M4 & 0.7111 & 0.6872 & 0.7350 & 0.0531 & $0.0009^{*}$ \\
\hline \multirow[t]{4}{*}{ ASCVD, borderline } & M1 & 0.9021 & 0.8852 & 0.9191 & - & - \\
\hline & M2 & 0.9368 & 0.9228 & 0.9508 & $<0.0001^{*}$ & $<0.0001^{*}$ \\
\hline & M3 & 0.9389 & 0.9253 & 0.9526 & 0.1197 & $0.0116^{*}$ \\
\hline & M4 & 0.9436 & 0.9307 & 0.9565 & $0.0087^{*}$ & $0.0011^{*}$ \\
\hline \multicolumn{7}{|l|}{ LASSO } \\
\hline \multirow[t]{4}{*}{ Obesity } & M1 & 0.5654 & 0.5052 & 0.6256 & - & - \\
\hline & M2 & 0.5654 & 0.5052 & 0.6256 & 1.0000 & 1.0000 \\
\hline & M3 & 0.5654 & 0.5052 & 0.6256 & 1.0000 & 1.0000 \\
\hline & M4 & 0.5896 & 0.5305 & 0.6487 & 0.1301 & $0.0038^{*}$ \\
\hline \multirow[t]{4}{*}{ Hypertension, stage-1 } & M1 & 0.6358 & 0.5778 & 0.6938 & - & - \\
\hline & M2 & 0.6749 & 0.6189 & 0.7308 & $0.0230^{*}$ & $<0.0001^{*}$ \\
\hline & M3 & 0.6770 & 0.6212 & 0.7329 & 0.6437 & 0.0546 \\
\hline & M4 & 0.6806 & 0.6250 & 0.7362 & 0.3969 & $0.0050^{*}$ \\
\hline \multirow[t]{4}{*}{ ASCVD, borderline } & M1 & 0.8880 & 0.8458 & 0.9303 & - & - \\
\hline & $M 2$ & 0.9505 & 0.9259 & 0.9751 & $<0.0001^{*}$ & $<0.0001^{*}$ \\
\hline & M3 & 0.9486 & 0.9234 & 0.9738 & 0.1339 & 0.0583 \\
\hline & M4 & 0.9488 & 0.9241 & 0.9736 & 0.9281 & $0.0170^{*}$ \\
\hline
\end{tabular}

${ }^{\text {a Compared with previous model }}$

${ }^{*} P<0.05$ 


\section{Discussion}

Assessing and responding to social needs is a major priority for health care systems seeking to deliver high-value care and improve population health. Efforts to better integrate these activities into routine clinical encounters and standard of care [6] include social needs EHR documentation strategies [33], innovative care models [34, 35], and cross-sector collaboration [36]. This study builds on the existing literature by evaluating the relationship between responses to a standardized social needs assessment and accepted measures of cardiometabolic health outcomes. Our intention is to highlight practical analytical tools for leveraging social needs information from PRAPARE and similar screening tools [37, 38] to better understand the association between social needs and commonly-studied cardiometabolic outcomes. The application of these analytical tools has the potential to enhance value-based care, population health management, panel management [13], and integrated socialmedical care model design and implementation $[39,40]$.

We evaluated the relationship between data from PRAPARE and three cardiometabolic outcomes using two predictive analytic approaches. We found that social needs were more prevalent in patients with hypertension and borderline-or-higher ASCVD risk. Interestingly, social needs were less prevalent in obese patients compared to those who were not obese. Lack of housing, high stress, and access to medicine and health care were the only social needs that were selected in models across more than one clinical risk outcome. These social needs may be proxies for additional, interrelated non-medical drivers of health and do not represent causal mechanisms between social needs and clinical outcomes.

The presence of social needs was associated with lower prevalence of obesity. Existing literature indicates that this counter-intuitive finding may be because obesity has a unique and multifactorial relationship to social needs and $\mathrm{SDOH}$ that varies by cultural context, race, ethnicity, and gender [41]. This finding highlights the importance of both understanding that associations between social needs and clinical outcomes depend on how adverse outcomes are defined, as well as the need for cautious interpretation of the directionality of the effect based on the variables retained in the model.

We hypothesized that the predictive analytic approaches would demonstrate moderate performance for all three cardiometabolic outcomes (c-statistic > 0.65); we found support for this hypothesis for ASCVD risk and hypertension, but not for obesity. We noted that model performance for ASCVD risk was very high even without including the clinical parameters or health behaviors used to calculate an ASCVD risk score as predictor variables (blood pressure, total and high density lipoprotein cholesterol, diabetes diagnosis, smoking status, and hypertension treatment). The comparison across nested models further contextualizes this finding. The inclusion of social needs variables for Model 4 resulted in a statistically significant increase in prediction performance compared to Model 3 in the backwards stepwise approach. The small decrease in the LASSO approach performance between the same models may be due to overfitting on a small sample, despite regularization to prevent it, and limitations to increases to prediction performance with increasingly granular data on an individual's unmet social needs. Nevertheless, this still suggests that social needs assessment data as a whole has useful associations with clinical outcomes in the absence of information on health behaviors and biometric data.

We found that the stepwise logistic and machine learning LASSO regression models demonstrated similar performance, a finding consistent with prior studies assessing the performance of predictive models [42, 43]. A potential explanation is that the advantages of more advanced, resource-intensive machine learning techniques like LASSO regression or random forest models, compared to stepwise logistic regression models, require more observations and dimensionality to become apparent [31]. The number and functional form of included variables also can influence results, with similar research demonstrating better performance for machine learning approaches when more variables and continuous variables are used [44]. This underscores the importance of considerations regarding data transformation, variable functional form, and data missingness when using and selecting a predictive analytical approach.

This study has several limitations. Because PRAPARE was administered only to a subset of patients referred to behavioral health, there was less variation in social need levels to base predictive analytics. The smaller sample size of patients from multiple clinics within a FQHC may have limited prediction performance and generalizability. Generalizability may also be limited by the setting-FQHCs in the southeastern United States. Finally, the prediction modeling approaches used in this analysis do not allow for making conclusions on potential causal inference. Despite these limitations, this study contributes to an emerging evidence base that suggests the formal and pragmatic validity of PRAPARE and provides insights into how social needs data could be used in outpatient settings to predict cardiometabolic health outcomes.

Predictive analytics may have the potential to proactively identify patients at higher risk for poor health outcomes who could benefit from an intervention, even in the absence of data obtained in current screening methods for cardiometabolic outcomes; our results align 
with previous literature suggesting limits to the utility of SDOH data for risk prediction [45]. In other words, additional SDOH data do not always lead to statistically significant improvements in prediction performance. This is relevant as payors, including state Medicaid programs [46], collect social needs data for new enrollees to identify patients at risk for worsening medical complexity based on social needs in order to improve population health. Our findings suggest that performance may depend on how clinical outcomes are defined and that relationships between social needs assessment data and outcomes vary by disease pathway.

\section{Future research directions}

Future research should evaluate social needs prevalence and association with additional clinical outcomes, using prospective data to understand how social needs data can be used to predict clinical risk and, ultimately, improve population health. Though this study focused on moderate clinical outcomes that may be useful for proactive intervention, outcomes that correspond to more severe clinical conditions (e.g., A1c $>8.0 \%$, ASCVD $>20 \%$ ) may have differing and perhaps stronger associations with unmet social needs. Ideally, this would include linking multiple data sources to comprehensively describe patient behaviors and environment in addition to information on social needs to predict other clinical risk and health status indicators including uncontrolled diabetes, co-morbidity burden, and behavioral health outcomes. Moreover, risk prediction around social needs will only add significant value if it is coupled with implementing evidence based responses to social needs that meaningfully address social needs to improve health outcomes in a cost-effective manner. These responses, or social care interventions, will need to be rigorously tested in diverse settings among a study sample of sufficient size to detect its impact on outcomes of interest including medication adherence, utilization, and cardiovascular health status.

Understanding the relationship between clinical outcomes and social needs may have important ramifications for how payers adjust for risk. In addition, future research should also evaluate the relationship between social needs assessment data and the likelihood of requiring costly types of heath care utilization including inpatient and emergency department visits. As social need screening becomes wider spread, there is a need to understand how this data can be used to improve health equity as health systems focus on improving population health. For example, our findings and future research can inform the business case for health systems to implement interventions to address social needs, which has the potential to narrow disparities in care resulting from social and economic inequities [47]. A critical step will be to design quality measures that complement care guidelines to focus support on medically vulnerable patients with unmet social needs [48].

\section{Conclusions}

Associations between standardized social needs assessment data and clinical outcomes vary by cardiometabolic outcome and may depend on how clinical outcomes are defined, which has implications for designing population health management and quality improvement initiatives using social needs assessment data on a defined patient population. Predictive analytics has the potential to leverage associations between clinical outcomes and social needs assessment data; however, there are limits to the utility of social needs data for improving predictive performance. Future research should further evaluate the utility of social needs assessment data to predict forms of clinical risk and health care utilization, especially as this data becomes more available in administrative or health records.

\section{Abbreviations \\ AIC: Akaike information criterion; ASCVD: Atherosclerotic cardiovascular disease; BIC: Bayesian information criterion; BMI: Body mass index; EHR: \\ Electronic health record; FQHC: Federally qualified health center; LASSO: Least absolute shrinkage and selection operator; OR: Odds ratio; PRAPARE: Protocol for responding to and assessing patients'assets, risks, and experiences; SDOH: Social determinants of health.}

\section{Supplementary Information}

The online version contains supplementary material available at https://doi. org/10.1186/s12872-021-02149-5.

Additional file 1. Comparison across LASSO logistic regressions across the three clinical outcomes. Models and c-statistics for cross-validation, minimum-AIC, minimum-BIC, and adaptive LASSO models.

Additional file 2. Included covariates for logistic nested models. Description of four nested models for stepwise logistic and LASSO approaches.

\section{Acknowledgements}

We gratefully acknowledge Lawrence Trachtman, Carolyn Crowder, and colleagues at the Lincoln Community Health Center for their support of this work. In addition, we thank the Duke University Health System's Analytics Center of Excellence for data collection and analytical support.

\section{Authors' contributions}

$C D, C S, J T, D E, K R, M W$, and $H E$ contributed to the study conception and design. CD, JT, HE, and CS participated in decisions related to data collection. $\mathrm{CD}, \mathrm{TL}$, and JT contributed to data analysis. The initial drafts of the manuscript were prepared by CD with feedback on the interpretation of the results from all authors. All authors read and approved the final manuscript.

\section{Funding}

This study was supported in part by the BlueCross BlueShield of North Carolina Foundation and by the National Heart, Lung, And Blood Institute of the National Institutes of Health under Award Number K12HL138030.

\section{Availability of data and materials}

The data that support the findings of this study are available upon reasonable request from the corresponding author $C D$. The data are not publicly available 
due to them containing information that could compromise research participant privacy

\section{Declarations}

\section{Ethics approval and consent to participate}

This protocol was reviewed and determined to be exempt by the Duke University Health System Institutional Review Board. The need for consent was waived. The participating FQHC granted permission to allow access to raw, de-identified data through a signed collaboration agreement with Duke University.

\section{Consent for publication}

Not applicable.

\section{Competing interests}

The authors declare that they have no competing interests.

\section{Author details}

'Department of Population Health Sciences, Duke University School of Medicine, 215 Morris Street, Durham, NC 27701, USA. ${ }^{2}$ Department of Health Policy and Management, Gillings School of Global Public Health, University of North Carolina at Chapel Hill, 135 Dauer Dr, Chapel Hill, NC 27519, USA. ${ }^{3}$ Department of Medicine, Duke University School of Medicine, 2301 Erwin Rd, Durham, NC 27705, USA. ${ }^{4}$ Durham VA Healthcare System, 508 Fulton St, Durham, NC 27705, USA. ${ }^{5}$ Lincoln Community Health Center, 1301 Fayetteville St, Durham, NC 27707, USA. ${ }^{6}$ Department of Family Medicine and Community Health, Duke University School of Medicine, DUMC 2914, Durham, NC 27710, USA.

Received: 17 January 2021 Accepted: 6 July 2021

Published online: 14 July 2021

\section{References}

1. Marmot M. Economic and social determinants of disease. Bull World Health Organ. 2001;79:988-9.

2. Booske BC, Athens JK, Kindig DA, Park H, Remington PL. Different perspectives for assigning weights to determinants of health. Madison: University of Wisconsin: Population Health Institute; 2010.

3. Beck AF, Cohen AJ, Colvin JD, Fichtenberg CM, Fleegler EW, Garg A, et al. Perspectives from the society for pediatric research: interventions targeting social needs in pediatric clinical care. Pediatr Res. 2018;84(1):10.

4. Alley DE, Asomugha CN, Conway PH, Sanghavi DM. Accountable health communities - addressing social needs through Medicare and Medicaid. N Engl J Med. 2016;374(1):8-11.

5. Gottlieb L, Tobey R, Cantor J, Hessler D, Adler NE. Integrating social and medical data to improve population health: opportunities and barriers. Health Aff. 2016;35(11):2116-23.

6. Fraze TK, Brewster AL, Lewis VA, Beidler LB, Murray GF, Colla CH. Prevalence of screening for food insecurity, housing instability, utility needs, transportation needs, and interpersonal violence by US physician practices and hospitals. JAMA Netw Open. 2019;2(9):1911514.

7. Moore J, Adams C, Tuck K. Medicaid access and coverage to care in 2018: results from the Institute for Medicaid Innovation's 2019 annual Medicaid managed care survey. 2018.

8. Weir RC, Proser M, Jester M, Li V, Hood-Ronick CM, Gurewich D. Collecting social determinants of health data in the clinical setting: findings from national PRAPARE implementation. J Health Care Poor Underserved. 2020;31(2):1018-35.

9. National Association of Community Health Centers AoAPCHO, Association OPC. PRAPARE implementation and action toolkit. Author Bethesda, MD; 2016.

10. Weir CR, Jester M. Assessing the relationship between social determinants of health and outcomes: findings from the PRAPARE pilot. nachc. org: National Association of Community Health Centers; 2018 June 25, 2018.
11. Henrikson NB, Blasi PR, Dorsey CN, Mettert KD, Nguyen MB, WalshBailey $C$, et al. Psychometric and pragmatic properties of social risk screening tools: a systematic review. Am J Prev Med. 2019;57(6):S13-24

12. Cantor J, Cohen L, Mikkelsen L, Pañares R, Srikantharajah J, Valdovinos E. Community-centered health homes. Oakland: Prevention Institute; 2011.

13. Neuwirth EEB, Schmittdiel JA, Tallman K, Bellows J. Understanding panel management: a comparative study of an emerging approach to population care. Perm J. 2007;11(3):12.

14. National Academies of Sciences $E$, Medicine. Accounting for social risk factors in Medicare payment: criteria, factors, and methods: National Academies Press; 2016.

15. Joynt KE, De Lew N, Sheingold SH, Conway PH, Goodrich K, Epstein AM Should Medicare value-based purchasing take social risk into account? N Engl J Med. 2017;376(6):510-3.

16. Virani SS, Alonso A, Benjamin EJ, Bittencourt MS, Callaway CW, Carson AP, et al. Heart disease and stroke statistics-2020 update: a report from the American Heart Association. Circulation. 2020;141(9):e139-596.

17. Drake C, Eisenson $\mathrm{H}$. Assessing and addressing social needs in primary care. N Engl J Med Catal. 2019;5(6):1-12.

18. PRAPARE Validation Using 8 "Gold Standard" Stages of Measure Development: National Association of Community Health Centers; 2019 [July 3, 2020]. http://www.nachc.org/wp-content/uploads/2019/10/prapare_ validation-fact-sheet-2019-9-26.pdf.

19. People H. Healthy people 2020 objectives. US Department of Health and Human Services. 2020.

20. Hedis N. Healthcare effectiveness data and information set. Washington: NCQA; 2009.

21. Kreatsoulas C, Anand SS. The impact of social determinants on cardiovascular disease. Can J Cardiol. 2010;26:8C-13C.

22. Whelton P, Carey R, Aronow W, Casey D Jr, Collins K, Himmelfarb DC, et al. 2017 ACC/AHA/AAPA/ABC/ACPM/AGS/APhA/ASH/ASPC/NMA/PCNA guideline for the prevention, detection, evaluation, and management of high blood pressure in adults: executive summary: a report of the American college of cardiology/American heart association task force on clinical practice guidelines (vol 71, pg 2199, 2018). J Am Coll Cardiol. 2018;71(19):2273-5.

23. Lloyd-Jones DM, Huffman MD, Karmali KN, Sanghavi DM, Wright JS, Pelser C, et al. Estimating longitudinal risks and benefits from cardiovascular preventive therapies among medicare patients: the Million Hearts Longitudinal ASCVD Risk Assessment Tool: a special report from the American Heart Association and American College of Cardiology. J Am Coll Cardiol. 2017;69(12):1617-36

24. Karmali KN, Goff DC, Ning H, Lloyd-Jones DM. A systematic examination of the 2013 ACC/AHA pooled cohort risk assessment tool for atherosclerotic cardiovascular disease. J Am Coll Cardiol. 2014;64(10):959-68.

25. Wells BJ, Chagin KM, Nowacki AS, Kattan MW. Strategies for handling missing data in electronic health record derived data. Egems. 2013;1(3):1035.

26. Hosmer DW, Lemeshow S. Applied logistic regression. New York: Wiley; 2000.

27. Heinze G, Wallisch C, Dunkler D. Variable selection-a review and recommendations for the practicing statistician. Biom J. 2018;60(3):431-49.

28. Ahrens A, Hansen CB, Schaffer ME. lassopack: model selection and prediction with regularized regression in Stata. arXiv:190105397. 2019.

29. Kennedy P. A guide to econometrics. Cambridge: MIT press; 2003.

30. DeLong ER, DeLong DM, Clarke-Pearson DL. Comparing the areas under two or more correlated receiver operating characteristic curves: a nonparametric approach. Biometrics. 1988;44:837-45.

31. Steyerberg EW, Harrell FE Jr, Borsboom GJ, Eijkemans M, Vergouwe Y, Habbema JDF. Internal validation of predictive models: efficiency of some procedures for logistic regression analysis. J Clin Epidemiol. 2001;54(8):774-81.

32. StataCorp L. Stata statistical software: release 16. College Station, TX. 2019.

33. Gold R, Bunce A, Cowburn S, Dambrun K, Dearing M, Middendorf M, et al. Adoption of social determinants of health EHR tools by community health centers. Ann Fam Med. 2018;16(5):399-407.

34. Theiss J, Regenstein M. Facing the need: screening practices for the social determinants of health. J Law Med Ethics. 2017;45(3):431-41. 
35. Doyle SK, Chang AM, Levy P, Rising KL. Achieving health equity in hypertension management through addressing the social determinants of health. Curr Hypertens Rep. 2019;21(8):58.

36. Byhoff E, Taylor LA. Massachusetts community-based organization perspectives on Medicaid redesign. Am J Prev Med. 2019;57(6):S74-81.

37. Billioux A, Verlander K, Anthony S, Alley D. Standardized screening for health-related social needs in clinical settings: the accountable health communities screening tool. NAM Perspectives. 2017.

38. Thomas-Henkel C, Schulman M. Screening for social determinants of health in populations with complex needs: implementation considerations. Available from: The Robert Wood Johnson Foundation and the Center for Health Care Strategies, New York, NY. 2017.

39. Garg A, Toy S, Tripodis Y, Silverstein M, Freeman E. Addressing social determinants of health at well child care visits: a cluster RCT. Pediatrics. 2015;135(2):e296-304.

40. Gottlieb LM, Wing $H$, Adler NE. A systematic review of interventions on patients' social and economic needs. Am J Prev Med. 2017;53(5):719-29.

41. Bennett GG, Wolin KY, Duncan DT. Social determinants of obesity. In: Obesity epidemiology: methods and applications. 2008. pp. 342-76.

42. Frizzell JD, Liang L, Schulte PJ, Yancy CW, Heidenreich PA, Hernandez AF, et al. Prediction of 30-day all-cause readmissions in patients hospitalized for heart failure: comparison of machine learning and other statistical approaches. JAMA Cardiol. 2017;2(2):204-9.
43. Miller PE, Pawar S, Vaccaro B, McCullough M, Rao P, Ghosh R, et al. Predictive abilities of machine learning techniques may be limited by dataset characteristics: insights from the UNOS database. J Cardiac Fail. 2019;25(6):479-83.

44. van der Ploeg T, Steyerberg EW. Feature selection and validated predictive performance in the domain of Legionella pneumophila: a comparative study. BMC Res Notes. 2016;9(1):1-7.

45. Chen M, Tan X, Padman R. Social determinants of health in electronic health records and their impact on analysis and risk prediction: a systematic review. J Am Med Inform Assoc. 2020;27(11):1764-73.

46. Cohen MK. North Carolina's transformation to Medicaid managed care. N C Med J. 2019;80(5):277-9.

47. Bachrach D. Addressing patients' social needs: an emerging business case for provider investment: Commonwealth Fund; 2014.

48. Anderson AC, O'Rourke E, Chin MH, Ponce NA, Bernheim SM, Burstin H. Promoting health equity and eliminating disparities through performance measurement and payment. Health Aff. 2018;37(3):371-7.

\section{Publisher's Note}

Springer Nature remains neutral with regard to jurisdictional claims in published maps and institutional affiliations.
Ready to submit your research? Choose BMC and benefit from:

- fast, convenient online submission

- thorough peer review by experienced researchers in your field

- rapid publication on acceptance

- support for research data, including large and complex data types

- gold Open Access which fosters wider collaboration and increased citations

- maximum visibility for your research: over 100M website views per year

At BMC, research is always in progress.

Learn more biomedcentral.com/submissions 